\title{
Tumor Laterality Midline
}

National Cancer Institute

\section{Source}

National Cancer Institute. Tumor Laterality Midline. NCI Thesaurus. Code C162614.

A finding indicating the tumor location is on the midline of the specimen. 